Journal for ImmunoTherapy of Cancer

\title{
PD-L1 targeting and subclonal immune escape mediated by PD-L1 mutations in metastatic colorectal cancer
}

\author{
Alexander Stein, ${ }^{1,2}$ Donjete Simnica (1) , ${ }^{3}$ Christoph Schultheiß (D) , ${ }^{3}$ \\ Rebekka Scholz (D) , ${ }^{3}$ Joseph Tintelnot, ${ }^{2}$ Eray Gökkurt, ${ }^{1}$ Lisa von Wenserski, ${ }^{3}$ \\ Edith Willscher, ${ }^{3}$ Lisa Paschold (1) , ${ }^{3}$ Markus Sauer, ${ }^{2}$ Sylvie Lorenzen, ${ }^{4}$ \\ Jorge Riera-Knorrenschild, ${ }^{5}$ Reinhard Depenbusch, ${ }^{6}$ Thomas J Ettrich, ${ }^{7}$ \\ Steffen Dörfel, ${ }^{8}$ Salah-Eddin Al-Batran, ${ }^{9}$ Meinolf Karthaus, ${ }^{10}$ Uwe Pelzer, ${ }^{11}$ \\ Lisa Waberer, ${ }^{12}$ Axel Hinke, ${ }^{13}$ Marcus Bauer, ${ }^{14}$ Chiara Massa, ${ }^{15}$ \\ Barbara Seliger (10 ${ }^{15}$ Claudia Wickenhauser, ${ }^{16}$ Carsten Bokemeyer, ${ }^{17}$ \\ Susanna Hegewisch-Becker, ${ }^{1}$ Mascha Binder (D) ${ }^{2,3}$
}

To cite: Stein A, Simnica D, Schultheiß C, et al. PD-L1 targeting and subclonal immune escape mediated by PD-L1 mutations in metastatic colorectal cancer. Journal for ImmunoTherapy of Cancer 2021;9:e002844. doi:10.1136/ jitc-2021-002844

- Additional supplemental material is published online only. To view, please visit the journal online (http://dx.doi.org/10. 1136/jitc-2021-002844).

AS, DS and CS contributed equally.

Accepted 08 July 2021
Check for updates

(C) Author(s) (or their employer(s)) 2021. Re-use permitted under CC BY-NC. No commercial re-use. See rights and permissions. Published by BMJ.

For numbered affiliations see end of article.

Correspondence to

Dr Mascha Binder;

mascha.binder@uk-halle.de

\section{ABSTRACT}

Background In patients with microsatellite stable (MSS) metastatic colorectal cancer (mCRC), immune checkpoint blockade is ineffective, and combinatorial approaches enhancing immunogenicity need exploration.

Methods We treated 43 patients with predominantly microsatellite stable $R A S / B R A F$ wild-type mCRC on a phase II trial combining chemotherapy with the epidermal growth factor receptor antibody cetuximab and the programmed cell death ligand 1 (PD-L1) antibody avelumab. We performed next-generation gene panel sequencing for mutational typing of tumors and liquid biopsy monitoring as well as digital droplet PCR to confirm individual mutations. Translational analyses included tissue immunohistochemistry, multispectral imaging and repertoire sequencing of tumor-infiltrating T cells. Detected PD-L1 mutations were mechanistically validated in CRISPR/Cas9-generated cell models using qRT-PCR, immunoblotting, flow cytometry, complement-dependent cytotoxicity assay, antibody-dependent cytotoxicity by natural killer cell degranulation assay and LDH release assay as well as live cell imaging of $\mathrm{T}$ cell mediated tumor cell killing.

Results Circulating tumor DNA showed rapid clearance in the majority of patients mirroring a high rate of early tumor shrinkage. In 3 of 13 patients expressing the high-affinity Fc $\gamma$ receptor $3 \mathrm{a}$ (Fc $\gamma \mathrm{R} 3 \mathrm{a}$ ), tumor subclones with $P D-L 1$ mutations were selected that led to loss of tumor PD-L1 by nonsense-mediated RNA decay in $P D-L 1 \mathrm{~K} 162 \mathrm{fs}$ and protein degradation in $P D-L 1$ L88S. As a consequence, avelumab binding and antibody-dependent cytotoxicity were impaired, while T cell killing of these variant clones was increased. Interestingly, $P D-L 1$ mutant subclones showed slow selection dynamics reversing on avelumab withdrawal and patients with such subclones had aboveaverage treatment benefit. This suggested that the $P D$ $L 1$ mutations mediated resistance to direct antitumor effects of avelumab, while at the same time loss of PD-L1 reduced biological fitness by enhanced $T$ cell killing limiting subclonal expansion.
Conclusion The addition of avelumab to standard treatment appeared feasible and safe. $P D-L 1$ mutations mediate subclonal immune escape to avelumab in some patients with mCRC expressing high-affinity FcyR3a, which may be a subset experiencing most selective pressure. Future trials evaluating the addition of avelumab to standard treatment in MSS mCRC are warranted especially in this patient subpopulation.

Trial registration number NCT03174405.

\section{INTRODUCTION}

In metastatic colorectal cancer (mCRC), the efficacy of immune checkpoint blockade (ICB) using programmed cell death protein 1 (PD-1)/PD-1 ligand 1 (PD-L1) targeting antibodies with or without cytotoxic $\mathrm{T}$ lymphocyte antigen 4 inhibitors has so far been limited to patients with high microsatellite instability (MSI-H) ${ }^{1-3}$ For the vast majority of patients with mCRC and microsatellite stable (MSS) tumors, immune checkpoint inhibitors have not yet shown relevant efficacy neither as single agent nor as maintenance in combination with fluoropyrimidines and Vascular endothelial growth factor (VEGF) antibody bevacizumab nor in combination with the MEK inhibitor cobimetinib. ${ }^{4-6}$ We hypothesized that the upfront combination of immunomodulatory chemotherapy (ie, 5-FU and oxaliplatin), immunogenic cell death inducing targeted therapy (ie, the EGFR antibody cetuximab) and ICB may be synergistic and sensitize toward the checkpoint inhibitor resulting in immune control and suppression of resistant tumor subclones. ${ }^{7-10}$ To this end, the AVETUX trial was designed to evaluate whether adding avelumab to FOLFOX and cetuximab is feasible and may show clinical 
or translational signs of synergistic efficacy. In contrast to other available PD-1/PD-L1 antibodies equipped with IgG4 or genetically engineered IgG1 constant regions lacking the ability to effectively bind complement or Fc gamma receptors (Fc $\gamma \mathrm{R})$, avelumab harbors a functional IgG1 domain suggested to induce both, checkpoint blockade as well as antibody dependent cytotoxicity (ADCC) when bound to the tumor cell surface. ${ }^{11}$ Thus, the combination of avelumab and cetuximab may be of particular interest in the immunosuppressive environment of MSS mCRC. To gain insights into the mode of action of this novel combination regimen as well as into resistance mechanisms and potential subgroups with superior clinical benefit, we designed an extensive translational program including comprehensive assessment of tumor biopsies prior to treatment and liquid biopsy monitoring of tumor subclones over treatment.

\section{MATERIAL AND METHODS Study design}

This is a single-arm, multicenter phase II trial designed to assess the combination of avelumab with cetuximab and mFOLFOX6 in patients with previously untreated $R A S / B R A F$ wild-type (wt), MSI independent mCRC. Main inclusion criteria were: $\geq 18$ years of age; Eastern Cooperative Oncology Group performance status 0 or 1; and no previous chemotherapy for metastatic disease (adjuvant chemotherapy allowed if terminated more than 6 months before trial start). The trial was conducted at 10 centers in Germany after approval by local ethics committees and competent authority and registered. All participants provided written informed consent. Patients or public were not involved in the design of the trial. The AVETUX regimen applied avelumab at a dose of $10 \mathrm{mg} / \mathrm{kg}$ intravenously over $60-90 \mathrm{~min}$ (biweekly from cycle 2 onward), cetuximab at a dose of $250 \mathrm{mg} / \mathrm{m}^{2}$ intravenously over 60-90 min (weekly, first dose $400 \mathrm{mg} / \mathrm{m}^{2}$ ) and a modified FOLFOX6 with oxaliplatin at a dose of $85 \mathrm{mg} / \mathrm{m}^{2}$ intravenously (day 1 ), 5 -FU $400 \mathrm{mg} / \mathrm{m}^{2}$ intravenously bolus (day 1 ) and 5 -FU $2400 \mathrm{mg} / \mathrm{m}^{2}$ intravenously continuous infusion (days 1-2), and LV at a dose of $400 \mathrm{mg} / \mathrm{m}^{2}$ intravenously. The primary endpoint was progression-free survival (PFS) rate at 12 months (according to RECIST V.1.1); secondary endpoints were overall response rate (ORR), early tumor shrinkage, progression-free and overall survival (OS) as well as toxicity. A sample size of 41 patients achieved $80 \%$ power to detect a positive signal of improvement by $17 \%$ compared with the PFS assumption for standard treatment at a one-sided alpha error level of 0.1 .

\section{Biomaterial}

Twenty mL peripheral blood (STRECK cell-free DNA BCT tubes) were obtained every 4-16 weeks for translational research. Paraffin-embedded tissue obtained during surgical removal or biopsy before treatment initiation as well as-if applicable-on treatment was collected.
Tissue immunohistochemistry and multispectral imaging Immunohistochemistry (IHC) for PD-L1 was performed with the Bond Polymer refine detection Kit (Leica) to deduce tumor proportion score (TPS) and immune cell (IC) score. As described elsewhere,${ }^{12}$ IHC was performed with antibodies directed against PanCK, CD3 and PD-L1 or CD56 and CD16, and multispectral imaging with antibodies against CD3, CD8, CD20, CD163, Foxp3 and PanCK (online supplemental table S1).

\section{Next-generation T cell receptor repertoire sequencing and data analysis}

Amplification of the $\mathrm{T}$ cell receptor beta chain (TRB) repertoire from circulating or tumor-infiltrating lymphocytes (TiLs) was done as described elsewhere. ${ }^{13-19}$ Sequencing and demultiplexing was performed on the Illumina MiSeq platform with $2 \times 301$ cycles at a coverage of 80000 reads per sample. Analysis of the TRB locus was computed using the MiXCR analysis tool V.3.0.5. ${ }^{20}$. Analyses were performed using $\mathrm{R}^{21}$ and the package tcR. ${ }^{22}$ Peripheral blood $\mathrm{T}$ cell diversification was calculated as follows: Shannon diversity index at week 4 minus Shannon diversity index at baseline. No delta or a negative delta were interpreted as not diversified, whereas a positive delta was interpreted as diversified.

\section{Next-generation gene panel sequencing}

For mutational profiling of tumor or circulating tumor DNA (ctDNA; liquid biopsy), up to $100 \mathrm{ng}$ of tumor Formalin-Fixed Paraffin-Embedded (FFPE) tissue DNA or ctDNA were used. Target genes with known pathogenic or resistance variants (cBio Cancer Genomics Portal ${ }^{23}$ ) as well as $P D-L 1$ were selected for the gene panel, and sequencing libraries were constructed using QIAseq Targeted DNA Custom Panels, Qiagen (online supplemental table S2). Sequencing was performed on the Illumina NextSeq or HiSeq platform with $2 \times 151$ cycles at a coverage of 26500 reads per target region. Variant calling of unique molecular identifier based data was performed using smCounter2 as described elsewhere. ${ }^{24}$ Tumors were classified as mutation positive, if the mutation was found in either tumor tissue or liquid biopsy at a variant allele frequency (VAF) exceeding $10 \%$ or if the mutation was concordantly found in tumor tissue and liquid biopsy independently of VAF. Follow-up liquid biopsies were classified mutation positive if the same mutation was found repeatedly in one patient independently of VAF. All variants that were found are listed in online supplemental table S3.

\section{Digital droplet PCR workflow and analysis}

ddPCR custom assays were designed by and ordered from IDT (Iowa, USA). To increase specificity locked nucleic acid bases were incorporated in the probes. Probes were HPLC purified and contained either a $5^{\prime}$-HEX (wildtype probe) or a $5^{\prime}$-FAM (mutant probe) reporter dye and a 3 ' Iowa Black Fluorescent quencher. Probe and primer sequences are listed in online supplemental table S4. 
Each reaction $(22 \mu \mathrm{L})$ contained $11 \mu \mathrm{L}$ 2xddPCR SuperMix for probes (no UTP) (Biorad), template DNA and primers and probes at a final concentration of $1.8 \mu \mathrm{M}$ and $500 \mathrm{nM}$, respectively. After droplet generation (AutoDG, Biorad), the plate was sealed with a pierceable foil heat seal (PX1 PCR plate sealer, Biorad), and the PCR was performed on a C1000 Touch thermal cycler (Biorad). The cycling conditions were as follows: $95^{\circ} \mathrm{C}$, $10 \mathrm{Min} ;\left[94^{\circ} \mathrm{C}, 30 \mathrm{~s} ; 59^{\circ} \mathrm{C}, 1 \mathrm{~min}\right] \times 45 ; 98^{\circ} \mathrm{C}, 10 \mathrm{~min} ; 12^{\circ} \mathrm{C}$, $30 \mathrm{~min} ; 4^{\circ} \mathrm{C}$ hold. Prior to the read-out on QX200 droplet reader (Biorad), the plate was left at room temperature for $10 \mathrm{~min}$.

ddPCR data were analyzed using the QX Manager 1.0 Standard Edition (Biorad).

Assay specificity was tested on gBlocks (IDT). Thresholds for positive/negative droplets were set manually for each assay according to the positive controls (gBlocks, IDT, online supplemental figure S1).

\section{Generation and evaluation of cell lines stably expressing patient-derived PD-L1 variants}

Novel $P D-L 1$ variants were functionally validated in five cell lines, including $\mathrm{Ba} / \mathrm{F} 3$ (PD-L1 negative murine pro B cell line), UT-SCC-14 (tongue squamous cell carcinoma), UT-SCC-29 (laryngeal squamous cell carcinoma), HT-29 (colorectal adenocarcinoma, KRAS wt) and DLD-1 (colorectal adenocarcinoma, KRAS G13D) (German Collection of Microorganisms and Cell Cultures $\mathrm{GmbH}$ ) after exclusion of Mycoplasma contamination. Endogenous PD-L1 was depleted using the CRISPR plasmid pU6 containing gRNAs complementary to nts 508-527 in exon 4 (gRNA1) and nts 339-358 in exon 3 (gRNA2) of PD-L1 (NM_014143.4) and verified by immunoblotting and flow cytometry. For ectopic re-expression of $P D-L 1$ variants, the wt coding sequence was cloned into the Lentiviral Gene Ontology (LeGO) vector LeGO-iC2Puro+via AsiSI/EcoRI. The pLeGO-PD-L1 wt construct was used as template to generate the PD-L1 L88S (c.263T $>$ C) by site-directed mutagenesis. The PD-L1 K162fs variant was synthesized and cloned into pLeGO-iC2-Puro+. Generation and transduction of lentiviral particles as well as selection of transduced cell was performed as described elsewhere. ${ }^{25}$ Cell lysates were generated using RIPA buffer supplemented with protease and phosphatase inhibitors (both Roche). Membrane fractions were generated using the ProteoExtract Native Membrane Protein Extraction Kit (Merck). Selective enrichment of membrane proteins was controlled via detection of prohibitin 1 and 2 (membrane) as well as NFkB subunit p65 (non-membrane) proteins (online supplemental table S1).

\section{Complement-dependent cytotoxicity (CDC)}

UT-SCC-14 cells were incubated with $2 \mu \mathrm{g}$ of avelumab and complement for 3 hours. Cetuximab in combination with matuzumab (1 $\mu \mathrm{g}$ each) served as positive control. Propidium iodine flow cytometry staining was used to analyze CDC.
Antibody-dependent cytotoxicity by natural killer (NK) cell degranulation assay and $\mathrm{LDH}$ release assay

NK cell degranulation was evaluated in co-culture assays with $P D-L 1$ variant cell lines by flow cytometry detection of CD107a. ${ }^{26} \mathrm{NK}$ cells from healthy volunteers were isolated via negative selection (RosetteSep Human NK Cell Enrichment Cocktail, Stemcell Technologies) and cultivated overnight in medium containing $1 \mathrm{ng} / \mathrm{mL}$ IL-15. NK cells were added to the respective target cells at 1:1 ratio in the presence of $1.2 \mu \mathrm{L}$ CD107a-PE-Cy7, $1 \mathrm{ng} /$

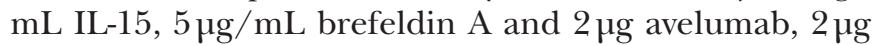
cetuximab or $2 \mu \mathrm{g}$ human IgG (Intratect) for 5 hours, washed and stained with anti-CD3-FITC/anti-CD56-PE (online supplemental table S1). NK cell-mediated target cell killing was also quantified using the Cytotoxicity Detection Kit (LDH) from Roche following the manufacturer's instructions.

\section{T cell mediated tumor cell killing}

$\mathrm{T}$ cell mediated tumor cell killing was assessed using the Incucyte Caspase-3/7 Reagent (Essen BioScience) according to the manufacturer's instructions. Untouched CD8+ T cells were isolated from pooled PBMCs of five healthy donors using the CD8+ TCell Isolation Kit (Miltenyi) and quantified using a Vi-CELL XR (Beckman Coulter). Five thousand HT-29 cells were seeded in triplicates in a 96-well flat bottom plate and cocultured with $50000 \mathrm{CD} 8+\mathrm{T}$ cells. For $\mathrm{T}$ cell activation, the medium was supplemented with $10 \mathrm{ng} / \mathrm{mL}$ recombinant IL-2 (BioLegend), $100 \mathrm{ng} / \mathrm{mL}$ anti-CD3 (BioLegend) and $100 \mathrm{ng} / \mathrm{mL}$ anti-CD28 (BioLegend) (online supplemental table S1). Read out of dead (=green-fluorescent) target cells was performed on an Incucyte S3 after 24 hours.

\section{qRT-PCR}

Total RNA was isolated using the Quick-RNA kit (Zymo Research) and reverse transcribed with SuperScript III (Thermo Fisher). Target amplification was performed on the CFX96 System (Bio-Rad) using the SYBR Select Master Mix CFX and primers for PD-L1 (forward: TGCTGAACGCATTTACTGTCAC; reverse: TCTGTCTGTAGCTA CTATGCTG), mCherry (forward: AGGA GGATAACATGGCCATCAT; reverse: ACCC TTGGTCACCTTCAGCT) and HPRT1 (forward: TGACACTG GCAAAACAATGCA; reverse: GGTCCTTTTCACCAGC AAGCT). The relative expression levels were normalized to HPRT 1 and calculated according to the comparative $\mathrm{Ct}$ $(\Delta \Delta \mathrm{Ct})$ method.

\section{Nonsense mediated mRNA decay (NMD)}

To assess the relevance of NMD for transcript turnover of the detected $P D-L 1$ variants, NMD was inhibited using $20 \mathrm{mM}$ caffeine for 4 hours. Selective enrichment of NMDtargeted $P D-L 1$ transcripts was detected (semi)quantitatively using (q)RT-PCR as described previously.

\section{PD-L1 degradation assays}

Cycloheximide (CHX) and tunicamycin (Tm) were obtained from Santa Cruz Biotechnology, Compound 
A

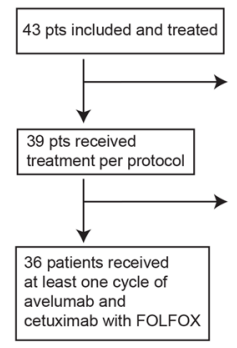

C

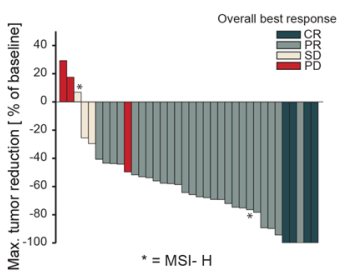

E

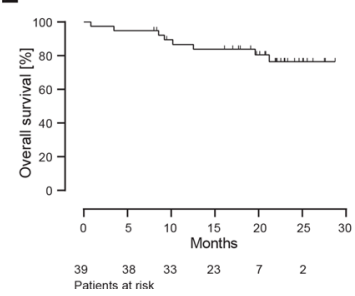

Figure 1 Efficacy of AVETUX protocol. (A) Consort diagram. (B) Kaplan-Meier estimates of progression-free survival. (C) Waterfall plot of best responses in target lesions (PD in responding patient was new lesion). (D) Spider plot depicting tumor measurements over time. Patients with MSI are marked with asterisk. (E) Kaplan-Meier estimates of overall survival. PD, progressive disease.

C/dorsomorphin and MG132 from Selleck Chem. To analyze degradation kinetics of PD-L1 variants, 2 million cells were seeded in 6-well format and either incubated with $100 \mu \mathrm{M}$ CHX for 3, 6 and 9 hours, with $10 \mu \mathrm{g} / \mathrm{mL} \mathrm{Tm}$ for 18 hours, with $10 \mu \mathrm{M}$ Compound C for 2 and 4 hours or with $20 \mu \mathrm{M}$ MG132 for 4 hours. Proteins were isolated as described previously and subjected to immunoblotting.

\section{RESULTS}

\section{Patient characteristics and study treatment}

Between June 2017 and July 2018, the AVETUX trial enrolled 43 patients, 41 thereof with MSS or MSI-low tumors. Patients 22 and 26 were MSI-high. Four patients were excluded after central tissue review with (mostly lower frequency) RAS (n=3) or BRAF mutations $(\mathrm{n}=1)$ (figure 1A). For eligible patients $(n=39)$, median age was 62 (range 29-82), 36 patients (92\%) had left sided primary tumors and 30 patients $(77 \%)$ had liver metastases. Prior adjuvant treatment rate was high with overall $31 \%(12 / 39)$ and oxaliplatin in 23\% (9/39) of patients. Patient characteristics are summarized in table 1.

Median follow-up was 21.2 months. The median number of cycles administered (at least one study component) was 18 cycles (range 1-35), with a median of 8 cycles
Table 1 Baseline characteristics of eligible patients

\begin{tabular}{|c|c|}
\hline \multirow[b]{2}{*}{ Characteristic } & \multirow{2}{*}{$\begin{array}{l}\text { AVETUX regimen } \\
\mathrm{n}=39\end{array}$} \\
\hline & \\
\hline \multicolumn{2}{|l|}{ Sex, no. (\%) } \\
\hline Male & $26(67)$ \\
\hline Female & $13(33)$ \\
\hline \multicolumn{2}{|l|}{ Age } \\
\hline Median years (range) & $62(29-82)$ \\
\hline \multicolumn{2}{|l|}{ ECOG performance status, n (\%) } \\
\hline 0 & $28(72)$ \\
\hline 1 & $11(28)$ \\
\hline \multicolumn{2}{|l|}{ Site of primary tumor, n (\%) } \\
\hline Left (splenic flexure-rectum) & $36(92)$ \\
\hline Right (transverse colon- caecum) & $3(8)$ \\
\hline \multicolumn{2}{|l|}{ Microsatellite status, n (\%) } \\
\hline MSI-H & $2(5)$ \\
\hline MSI-L & $1(3)$ \\
\hline MSS & $36(92)$ \\
\hline \multicolumn{2}{|l|}{ Prior adjuvant chemotherapy, n (\%) } \\
\hline Single agent fluoropyrimidine & $3(8)$ \\
\hline Oxaliplatin based & $9(23)$ \\
\hline \multicolumn{2}{|l|}{ Metastatic sites, n (\%) } \\
\hline Liver & $30(77)$ \\
\hline Lung & $12(31)$ \\
\hline Lymph node (distant) & $18(46)$ \\
\hline Bone & $3(8)$ \\
\hline Other soft tissue & $5(13)$ \\
\hline Ascites & $1(3)$ \\
\hline Pleural effusions & $1(3)$ \\
\hline Other & $7(18)$ \\
\hline
\end{tabular}

ECOG, Eastern Cooperative Oncology Group; MSI, microsatellite instability - high (H) or low (L); MSS, microsatellite stable.

of oxaliplatin, 12 of cetuximab and 16 of avelumab. The combination of avelumab and cetuximab was applied for a median time of 5.4 months.

\section{Clinical data: efficacy and safety}

The Kaplan-Meier estimation of PFS from the time point of protocol treatment start is shown in figure $1 \mathrm{~B}$, which is based on a total of 31 observed events $(79 \%)$ in the intention-to-treat population of 39 patients. The PFS rate at 12 months was $40 \%$ and the median PFS was 11.1 months (95\% CI 9.6 to 13.5 months). Based on the high rate of prior oxaliplatin $(23 \%)$ and early non-treatment related drop-outs, an exploratory analysis excluding these patients showed a PFS of 13.2 months. The overall response rate in the RECIST evaluable population $(\mathrm{n}=37)$ was $81 \%$ (95\% CI $65 \%$ to $92 \%$ ) (MSS/MSI-low $83 \%$ ) with four complete responses $(11 \%)$ and a disease control rate of $89 \%$ (figure 1C,D). Early tumor shrinkage of at least $20 \%$ 
Table 2 Grade $3 / 4$ adverse events occurring in $>5 \%$ of patients

\begin{tabular}{ll}
\hline Grade 3/4 adverse event (>5\%) & Incidence, $\mathbf{n}(\%)$ \\
\hline Infections & $12(32)$ \\
\hline Neutropenia & $12(32)$ \\
\hline Rash & $7(18)$ \\
\hline Peripheral sensory polyneuropathy & $5(13)$ \\
\hline Diarrhea & $4(11)$ \\
\hline Leukopenia & $4(11)$ \\
\hline Thromboembolic event & $4(11)$ \\
Vomiting & $3(8)$ \\
Fever & $3(8)$ \\
Allergic reactions & $3(8)$ \\
Syncope & $3(8)$ \\
Hypertension & $3(8)$ \\
Nausea & $2(5)$ \\
Abdominal pain & $2(5)$ \\
Sepsis & $2(5)$ \\
Lipase increased & $2(5)$ \\
Thrombocytopenia & $2(5)$ \\
\hline
\end{tabular}

during the first 8 weeks was observed in 30 patients $(81 \%)$. The current OS Kaplan-Meier curve estimates are shown in figure 1E, which plateau at $77 \%$ at a median follow-up of 21.2 months. Until the data cut-off in September 2019, only eight events occurred. Secondary resection of metastases was achieved in 6 out of 39 patients $(15 \%)$ of which five stopped treatment after surgery. Treatment-related adverse events are summarized in table 2, revealing no unexpected findings or overlapping toxicities.

\section{Immunological biomarkers}

Since ICB represented the experimental component of the treatment regimen, we studied potential immunological biomarkers of response.

We did not observe any association of PD-L1 TPS with PFS in our patient cohort (online supplemental figure S2). Yet, this analysis was hampered due to low tumor cell count ( $>30 \%$ of samples with less than 100 tumor cells), lack of the invasion front ( $>25 \%$ biopsy specimens $)^{27} 28$ (online supplemental figure S3) and discordant PD-L1 staining with different antibody clones in $15 \%$ of samples (online supplemental table S5).

Next, we explored blood-circulating and tumorinfiltrating $\mathrm{T}$ cells (TiLs) by next-generation immunosequencing to deduce potentially predictive immune signatures based on diversification (related to an increase in Shannon diversity index) of the peripheral blood T cell space. ${ }^{1329}{ }^{30}$ In contrast to prior data from monotherapy trials, our combinatorial approach did not reveal any association with PFS (online supplemental figure S4). Tissue deep sequencing revealed a broad range of $\mathrm{T}$ cell infiltration levels across the cohort (online supplemental figure
S5A). Since no defined threshold is available in literature, we defined $0.5 \%$ as cut-off for high $(\mathrm{n}=14)$ versus low $(n=28)$ TiL levels. Interestingly, four of five patients with febrile reactions to ICB belonged to the subset of patients with high levels of $\mathrm{T}$ cell infiltration, and high TiL levels showed a trend toward improved PFS $(\mathrm{p}=0.07$, online supplemental figure S5B). The TiL composition was rather heterogeneous both across the cohort and in different portions of the same tumor as analyzed by multispectral imaging with some patients showing CD8+ biases, some CD4+ biasesand others showing $\mathrm{T}$ cell infiltration biased toward Tregs (online supplemental figure S6).

\section{Liquid biopsy disease monitoring}

The complete study cohort was subjected to tumor tissue and liquid biopsy testing at baseline using a gene panel covering the most frequent driver and resistance mutations in mCRC (online supplemental table S2). In 40 out of 43 patients, mutations in the baseline tumor tissue and liquid biopsy screening were identified, which overlapped in 36 patients in at least one gene mutation (figure 2A,B). The vast majority of these patients turned negative or clearly reduced their circulating tumor DNA (ctDNA) levels to $<1 \%$ after 4 weeks of treatment (figure 2C). Next, serial liquid biopsies were assessed as a predictor of disease progression. Of 26 cases with ctDNA positivity and progressive disease during the observation period, reappearance or increase of ctDNA preceded disease progression by a median of 8.5 weeks (mean 13 weeks) in those who initially reduced their ctDNA levels to $<1 \%$ or turned ctDNA negative (figure 2D).

\section{Mutations in the MAPK signaling axis}

Beside baseline RAS mutations excluding the application of EGFR antibodies, recent studies on EGFR inhibition found high rates of acquired secondary resistance mediating downstream RAS mutations or EGFR ectodomain mutations disrupting the antibody's epitope. ${ }^{25}{ }^{31-36}$ While EGFR ectodomain mutations may appear in up to $20 \%$ of patients during EGFR directed treatment, acquired RAS mutations have been reported more frequently $(32 \%-$ $44 \%$ of cases). ${ }^{37-40}$ In our cohort, five patients showed (mainly low level) RAS mutations at baseline confirmatory central testing (patients 5, 12, 15, 34 and 42), leading to the post hoc exclusion due to violation of selection criteria in 2 patients ( $>10 \%$ mutant allels in tissue). Patients 5, 34 and 12 cleared their KRAS mutant ctDNA from blood in the course of treatment with patients 5 and 12 even achieving early CR (figure 2E). Patient 42's KRAS Q131H mutation (VAF 0.14\% in liquid biopsy at baseline) did not steadily expand on treatment but showed a rather fluctuating course not exceeding VAFs of $10 \%$ and the patient achieved early CR and long-term disease control (figure 2E). Patient 43 secondarily developed a classical NRAS mutation shortly after withdrawal of oxaliplatin. While the initial liquid biopsy was negative for NRAS Q61L, this mutation steadily increased on treatment, and within 7 weeks from first detection of this mutation, 

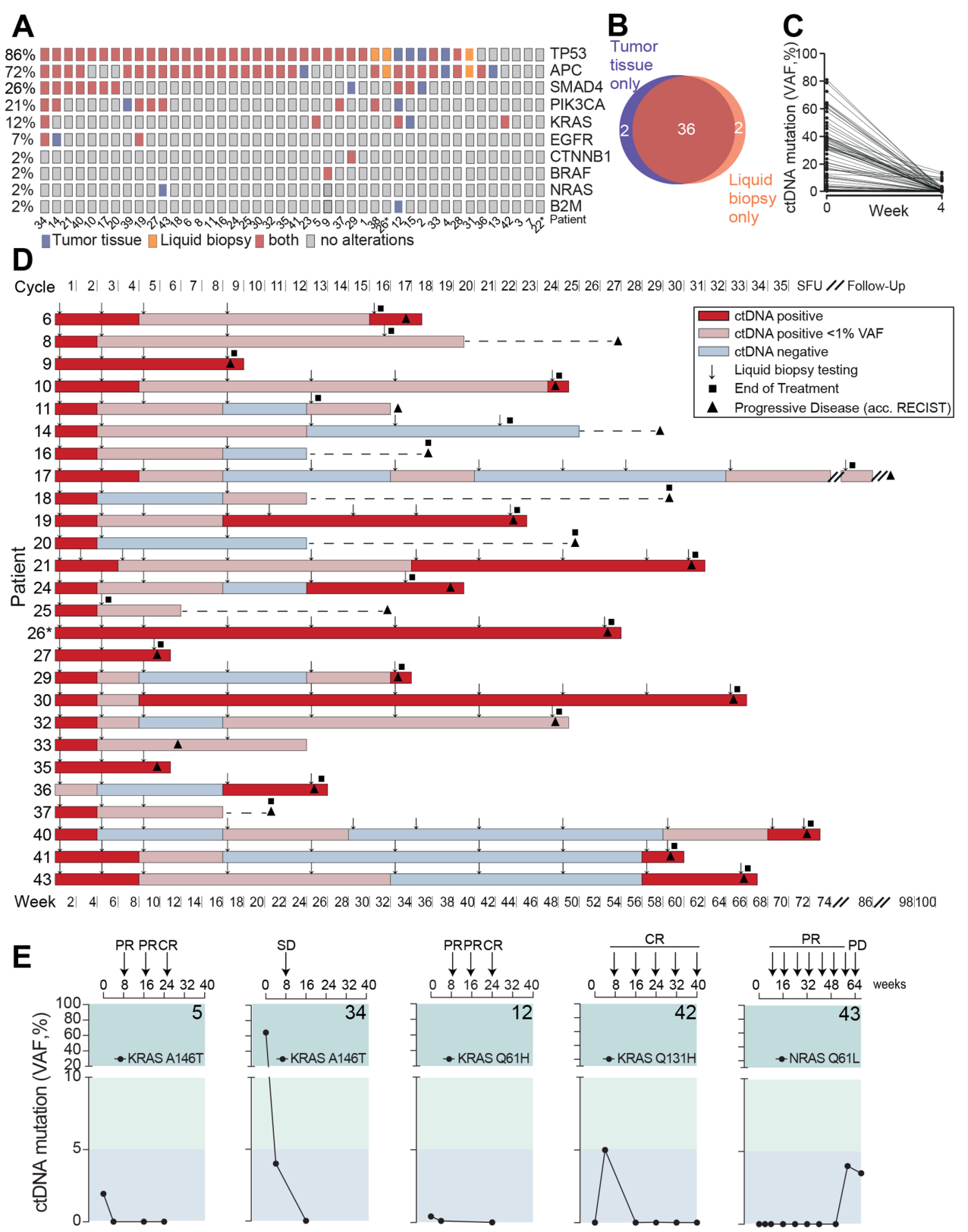

Figure 2 Mutational profiling and liquid biopsy disease monitoring. (A) Distribution of mutation spectra in FFPE tumor tissue and liquid biopsy at baseline evaluation. (B) Venn diagram of patients of which tumor driver mutations were detected by gene panel sequencing in FFPE tissue and/or liquid biopsy, respectively. (C) Circulating tumor (CT) DNA clearance from baseline to week 4 after treatment initiation. (D) Serial liquid biopsy testing in patients with disease progression during observational period. Gray box: increase or reappearance of ctDNA prior to clinical PD in weeks. Line indicates median. (E) KRAS and NRAS circulating tumor DNA monitoring during AVETUX therapy regimen. Respective patient number in bold. Patients with MSI are marked with asterisk. CR, complete response; FFPE, formalin-fixed paraffin-embedded; PR, partial response; PD, progressive disease; SD, stable disease; VAF, variant allele frequency; SFU, safety follow-up.

the patient progressed clinically (figure 2E). In line with the exceedingly low rate of emerging $R A S$ mutations (only one in 43 patients with emerging NRAS mutated ctDNA levels and progression), there was no evidence for acquired EGFR ectodomain mutations on this protocol.

\section{Selection and dynamics of tumor subclones with mutations in targets relevant for ICB}

To search for potentially emerging avelumab-resistant tumor subclones, we used a liquid biopsy panel including genes known to be involved in resistance ${ }^{41}(\beta 2$-microglobulin
(B2M), Janus kinase 1 (JAK1)). Moreover, proposing direct antitumor effects as mechanism of action of avelumab (ADCC), we covered the whole coding region of PD- $L 1$ to be able to detect potential escape variants that may evade avelumab binding by epitope disruption or impaired membrane expression. In addition, we included regions within the $F c y$ receptor $(F c \gamma R)$ genes with known single nucleotide polymorphisms (SNPs) associated with highaffinity toward IgG1 that have been described to allow more potent NK cell ADCG. ${ }^{11}$ 
A

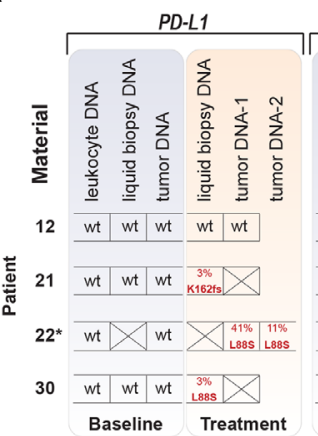

C

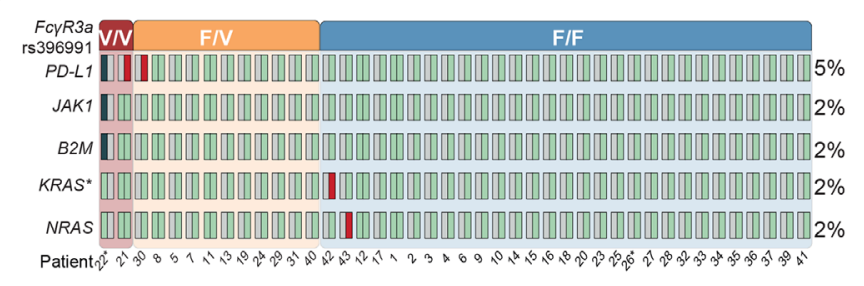

B
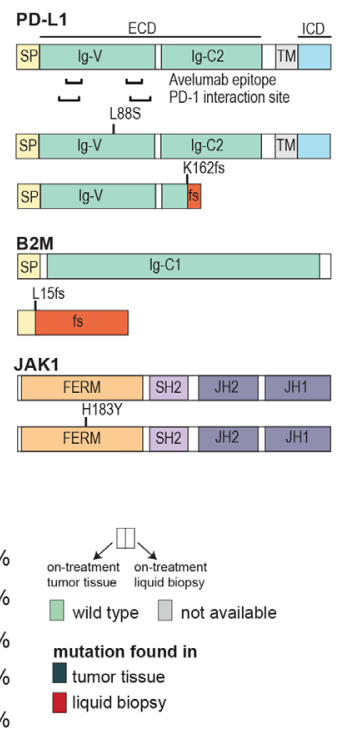

E

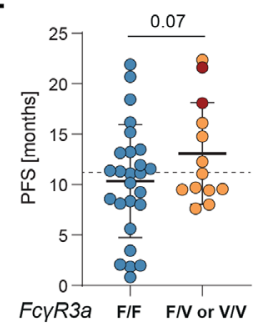

FCYR3a F/F F/V or V/V
$\mathbf{F}$

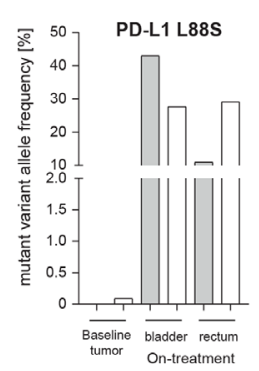

Patient $22^{*}$

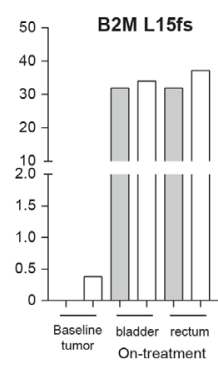

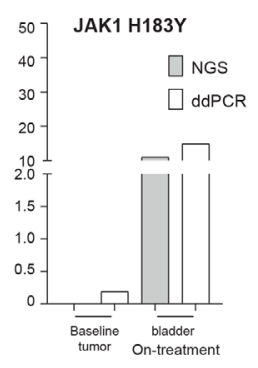

Figure 3 Selection of resistance variants on AVETUX protocol and clearance after avelumab withdrawal. (A) Overview of B2M, $J A K 1$ and $P D-L 1$ mutations in baseline and on-treatment tumor and liquid biopsy samples. Tumor samples collected under treatment or at EOT originate from: patient 12: liver metastasis, patient 22: tumor DNA 1: rectum and tumor DNA 2: bladder (same metastatic site as used for baseline testing), . (B) Localization of B2M, JAK1 and PD-L1 mutations. (C) Distribution of emerging resistance mutations to avelumab and cetuximab as well as $F c \gamma R 3 a$ genotype (rs396991) over the cohort. Asterisk on KRAS indicates that mutation disappeared in follow-up liquid biopsy samples. In total five on-treatment tumor samples were available. (D) ddPCR validation of immune checkpoint blockade resistance variants in patient 22. (E) PFS of patients with F/F versus F/N or V/V FcyR3a genotype (rs396991). Dotted line indicates median PFS of entire patient cohort. Statistical test: one-sided, unpaired t-test. (F) Longitudinal ctDNA and biopsy (hemicolectomy) mutational and CEA profile of patient 21 with a treatment-induced $P D-L 1 \mathrm{~K} 162 \mathrm{fs}$ mutation and patient 30 with a treatment-induced $P D-L 1$ L88S mutation. Treatment is indicated above each plot. Highlighted area indicates time during AVETUX regimen. Patients with MSI are marked with asterisk. APC, adenomatous polyposis coli; B2M, $\beta 2$-microglobulin; CEA, carcinoembryonic antigen; JAK1, Janus kinase 1; PD-L1, programmed cell death ligand 1; PFS, progression-free survival; PD, progressive disease; PR, partial response; TP53, tumor protein p53;

Indeed, NGS of ctDNA or on-treatment tumor samples $(\mathrm{n}=5)$ identified patients with mutations in the previously mentioned genes (figure 3A). Patient 22 showed both a B2M L15fs and a JAK1 H183Y mutation in on-treatment tumor biopsies. The B2M L15fs mutation was truncating, and the JAK1 H183Y was compatible with 
loss-of-functionality as in previously reported resistance variants (figure 3B). Interestingly, both on-treatment tumor samples (primary and metastasis) showed the $B 2 \mathrm{M}$ L15fs mutation, while the JAK1 H183Y mutation was only detectable in the metastasis and with a different VAF. In patient 12, a $B 2 M$ L15fs mutation was detectable by NGS already prior to treatment.

Moreover, we found two selected PD-L1 mutations in three patients of our cohort. Both mutations were located within the extracellular region of PD-L1, outside both the PD-L1/PD-1 interaction site and the avelumab epitope on PD-L1 (figure 3A,B). The PD-L1 L88S variant was a missense mutation, while the $P D-L 1$ K162fs mutation led to a frameshift with premature stop codon before the transmembrane domain. The truncated variant PD-L1 K162fs was found in patient 21 by liquid biopsy analysis with allele frequencies at the detection limit in the course of treatment. VAF increased substantially at disease progression suggesting that this mutation was involved in resistance to treatment (online supplemental table S3). Interestingly, the PD-L1 L88S mutation occurred in patient 22 who also showed B2M L15fs and JAK1 H183Y mutations. It was undetectable in the tumor tissue by NGS analysis before treatment initiation as well as in leukocyte DNA of this patient. In patient 30, the same missense mutation PD-L1 L88S was found at end of treatment at the time of disease progression. Distribution of all mutations selected on treatment are summarized in figure 3C.

We used ddPCR to validate our findings and could confirm all four variants using specific custom assays (online supplemental table S6 and online supplemental figure $\mathrm{S} 1$ ). Since this method is more sensitive than NGS, we exemplarily reanalyzed the baseline tumor samples of patient 22 who showed three ICB resistance mutations in on-treatment tumor tissue. Interestingly, while the baseline tumor sample of patient 22 was negative for these mutations by NGS, we identified small frequencies of the PD-L1 L88S, B2M L15fs and JAK1 H183Y variants by ddPCR suggesting the presence of these small subclones already at baseline and a subsequent selection of those clones on the AVETUX regimen (figure 3D).

Of note, patients 21 and 22 with $P D-L 1$ mutations were the only patients in the cohort homozygously expressing the V-allele of $F(\gamma R 3 a$ (rs396991) that is associated with high IgG1 affinity while patient 30 expressed the V-allele in a heterozygous fashion (figure 3C; online supplemental table S7). Association of the SNP with PD-L1 mutations as well as a trend toward improved PFS in patients homozygous or heterozygous for the V-allele $(\mathrm{p}=0.07$, figure $3 \mathrm{E})$ suggested that this may be a subset of patients experiencing strongest selective pressure by avelumab, in addition to the known higher susceptibility to cetuximab. ${ }^{42}$ In patients 21 and 30 , tumor material or ctDNA was available after progression on the AVETUX protocol several months after avelumab withdrawal. Interestingly, in both patients, PD-L1 mutant subclones (but not the tumor defining mutations) decreased in size or fully disappeared suggesting low biological fitness of these

\section{Patient 22}

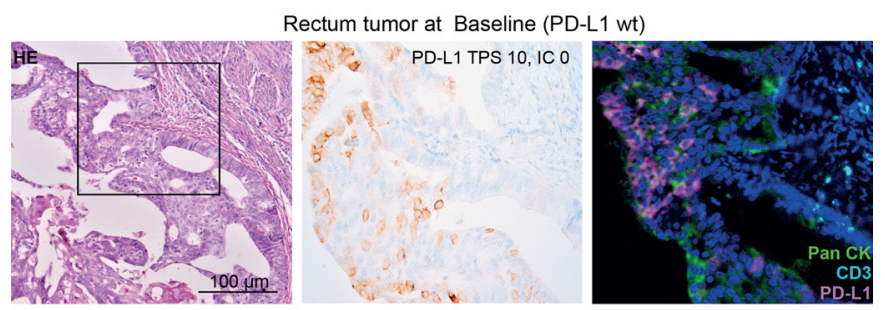

Rectum tumor 12 months on avelumab Tx (aquired PD-L1 L88S)

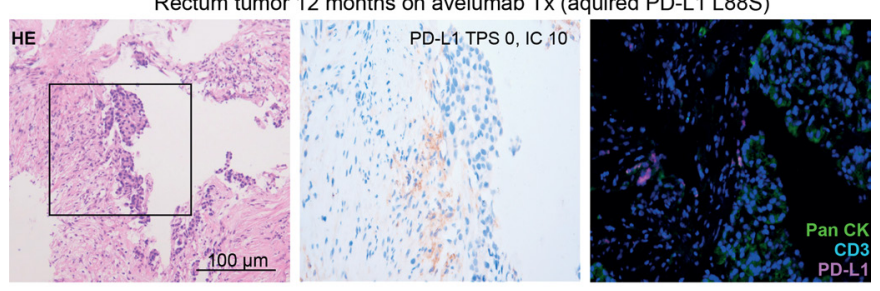

Bladder tumor 12 months on avelumab Tx (aquired PD-L1 L88S)

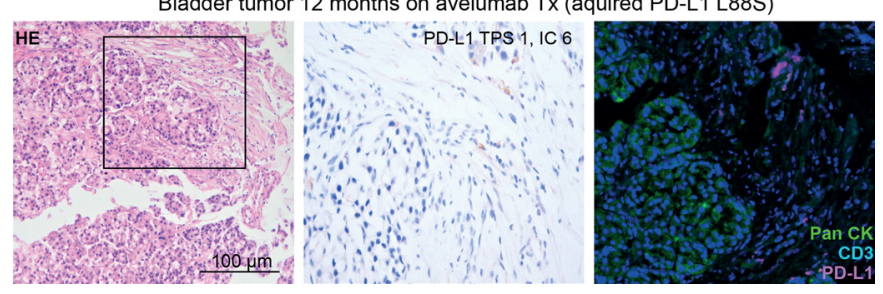

Figure $4 \mathrm{IHC}$ staining of PD-L1 in pre-treatment and ontreatment tumor tissue of patient 22 with genetic evidence of a treatment-induced $P D-L 1$ L88S mutation. Representative micrographs of H\&E and PD-L1 stainings of tumor tissue at $100 \times$ and $400 \times$ magnification, respectively. Moreover, multiplex IHC is shown using antibodies against PanCK (tumor cells, green), CD3 (T cells, turquoise) and PD-L1 (magenta). Nuclei are stained with DAPI. IC, immune cell score; IHC, immunohistochemistry; PD-L1, programmed cell death protein ligand 1; TPS, Tumor proportional score; Tx, treatment.

clones when the selective pressure exerted by avelumab was withdrawn (figure $3 \mathrm{~F}$ ).

Together, their selection on treatment, their localization outside the avelumab epitope and their association with the $F c \gamma R 3 a$ V-allele suggested that the identified $P D-L 1$ mutations may mediate immune escape toward avelumab through loss of the PD-L1 antigen on tumor cells. Since in patient 22 on-treatment tumor tissue was available, we performed PD-L1 immunohistochemistry (IHC) on this tissue and compared the results with pretreatment tumor tissue of the same patient (harboring only a minimal frequency of PD-L1 mutated clones). In line with our hypothesis, this patient showed a selective loss of PD-L1 surface staining on tumor cells while immune cells were clearly PD-L1 positive after avelumab exposure (figure 4). To definitively exclude epitope disruption by the mutation, we performed western blot analyses using clone QR1 used for tissue staining on cell lines strongly overexpressing PD-L1 L88S. This experiment confirmed that this antibody clone-recognizing an intracellular epitope of PD-L1-recognizes the PD-L1 L88S variant (online supplemental figure $\mathrm{S} 7$ ). 
Functional validation of novel PD-L1 mutations in cell models

To further explore the functional role of the detected $P D-L 1$ alterations, we generated five different cell models expressing these variants using CRSIPR/Cas9 and lentiviral transduction (figure 5A,B). Ectopic expression of $P D-L 1$ L88S led to a transcription-independent reduction of full-length glycosylated PD-L1 protein in subcellular membrane fractions as compared with the wt, while cell lines expressing the truncating K162fs mutation did not yield a PD-L1 signal on immunoblots (figure 5B-D; online supplemental figure S8). Consequently, PD-L1 L88S cells showed reduced, K162fs cells no avelumab surface binding, which was not attributable to epitope disruption (figure 5E; online supplemental figure S9).

In accordance with previous reports, ${ }^{44}$ CDC effector functions were not detectable (online supplemental figure S10). In contrast, cells expressing the L88S variant were markedly less able to induce degranulation of primary NK cells in the presence of avelumab as compared with the wt (figure $5 \mathrm{~F}$ ). The $100 \%$ positive staining rate of patient tumor material with antibodies against CD56 and/or CD16 clearly showed that all of the tumors were infiltrated with cells able to mediate ADCC under in vivo conditions (data not shown). PD-L1 K162fs transduced cells failed to mount an avelumab-mediated NK cell response (figure $5 \mathrm{~F}$ ). NK cell degranulation mediated by cetuximab was independent of PD-L1 status or variant (online supplemental figure S11). Direct LDH release as a measure for target cell killing confirmed this pattern (online supplemental figure S12).

To test whether the K162fs or L88S variants affect $\mathrm{T}$ cell suppression, we cocultured HT-29, DLD-1 and UT-SCC-14 cells expressing these variants with primary cytotoxic CD8+ T cells and monitored immune cell killing over time. While cells expressing wt $P D-L 1$ were more resistant to $\mathrm{T}$ cell mediated immune cell killing than L88S cells, cells expressing the K162fs variant were most effectively targeted by $\mathrm{T}$ cells (figure $5 \mathrm{G}$ ).

Further experiments were performed to explore the mechanisms underlying loss of PD-L1 membrane expression in these mutants. As demonstrated with inhibition experiments, the K162fs mRNA product is constantly degraded by the NMD machinery (online supplemental figure S13). Since reduced PD-L1 protein levels in cells expressing PD-L1 L88S were independent of transcription, we reasoned that this mutation had a lower stability and/or higher turnover as the wt. In accordance with this notion, PD-L1 L88S exhibited a shorter lifetime after blocking protein biosynthesis with cycloheximide (figure 6A,B) or $\mathrm{N}$-glycosylation (figure 6C), which is a key determinant of PD-L1 protein stability, ${ }^{45}$ with tunicamycin. Although the change from leucine to serine at position 88 might affect intrinsic protein stability, it also creates a potential phosphorylation site within the extracellular domain (ECD). Since homeostatic PD-L1 turnover includes phosphorylation-dependent degradation by the proteasome ${ }^{45}{ }^{46}$ we hypothesized that an additional phosphorylation site might enhance this process.
To test this, we focused on the PD-L1 interacting AMPactivated protein kinase (AMPK), which phosphorylates serines within the ECD of PD-L1. ${ }^{46}$ AMPK inhibition using compound $\mathrm{C}$ (dorsomorphin) caused a doubling of PD-L1 L88S protein levels, which was not observed when serine 88 was substituted with alanine (figure 6D). Notably, the turnover of L88A variant was identical to the wt (figure 6A-C). Blocking of the proteasome with MG132 causes an enrichment of the glycosylated and nonglycosylated PD-L1 variants that was substantially stronger in the PD-L1 L88S transduced cell lines (figure 6E). Taken together, our data suggest that the truncating mutation $P D-L 1$ K162fs is degraded by NMD, while the PD-L1 L88S variant creates a new phosphorylation site leading to loss of protein stability and proteasomal degradation.

\section{DISCUSSION}

We tested the combination of mFOLFOX6, cetuximab and avelumab in patients with $R A S / B R A F$ wt, predominantly MSS mCRC with a high rate of prior adjuvant chemotherapy. The combination was feasible showing a similar profile of adverse events as observed for cetux$\mathrm{imab} /$ chemotherapy combinations and ICB alone, except for some cases of unexpected fever episodes.

Despite the high ORR of $81 \%$, the median PFS of 11.1 months did not suggest significant additional benefit of the ICB component in the unselected cohort compared with historical data from other trials (median PFS ranging from 8.6 to 11.1 months). ${ }^{47-50}$ We therefore looked for subpopulations of patients with potentially increased treatment benefit. While baseline TiLs or T cell metrics were not predictive for treatment benefit, we found a trend toward longer PFS for patients expressing the $F \gamma R 3 a$ V-allele that promotes high-affinity receptor interactions that are known to enhance ADCC (as shown, for example, for cetuximab ${ }^{42}{ }^{43}$ ). This subset of patients seemed interesting in that we found some previously recognized ICB resistance mutations (in $B 2 M$ and $J A K$ genes $^{41}$ ) as well as-probably the most salient translational finding in this trial-PD-L1 mutations that abrogated avelumab binding in 3 of 13 patients ( 3 of 13 patients with the $F(\gamma R 3 \mathrm{SNP}$ and $3 / 43$ patients in total). Two different PD-L1 mutations were found: one represents a missense mutation at amino acid position 88 that leads to full loss of PD-L1 membrane positivity in on-treatment tumor tissue, but not in immune cells, supporting the view that this mutation was a genetic event selected exclusively in the tumor. Overexpression of PD-L1 L88S in our cell line models showed reduced levels of avelumab binding and activity but not full loss. This discrepancy was most likely due to the unphysiological level of overexpression of $P D$ L1 in the cell line models. Further experiments showed that loss of membrane PD-L1 on the cell surface in this mutant resulted from introduction of a new phosphorylation site, causing proteasomal degradation. The PD-L1 K162fs variant lacks a transmembrane domain due to a premature stop codon, its mRNA is degraded by NMD 
A

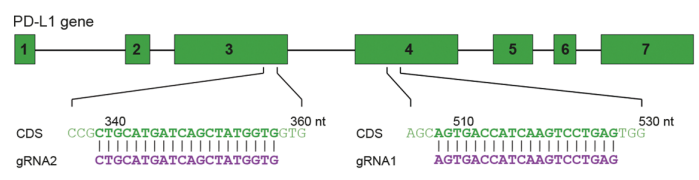

B

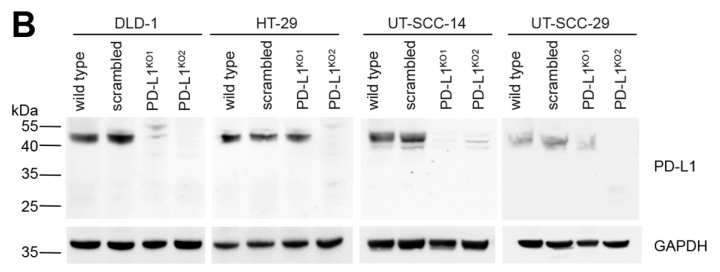

C
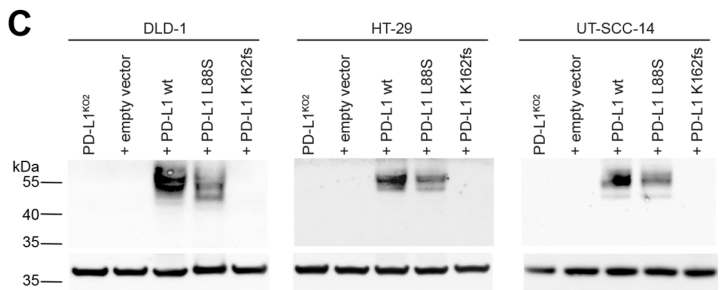

E
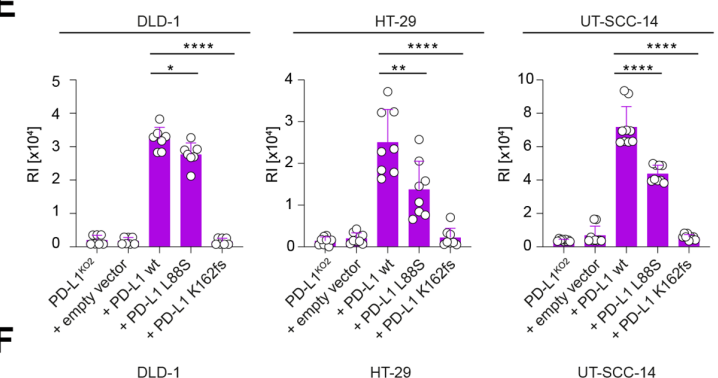

HT-29
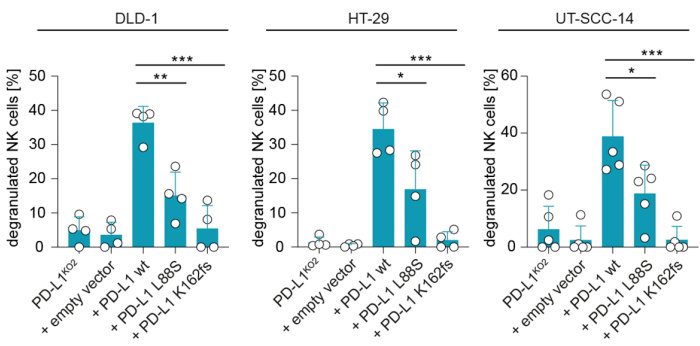

D
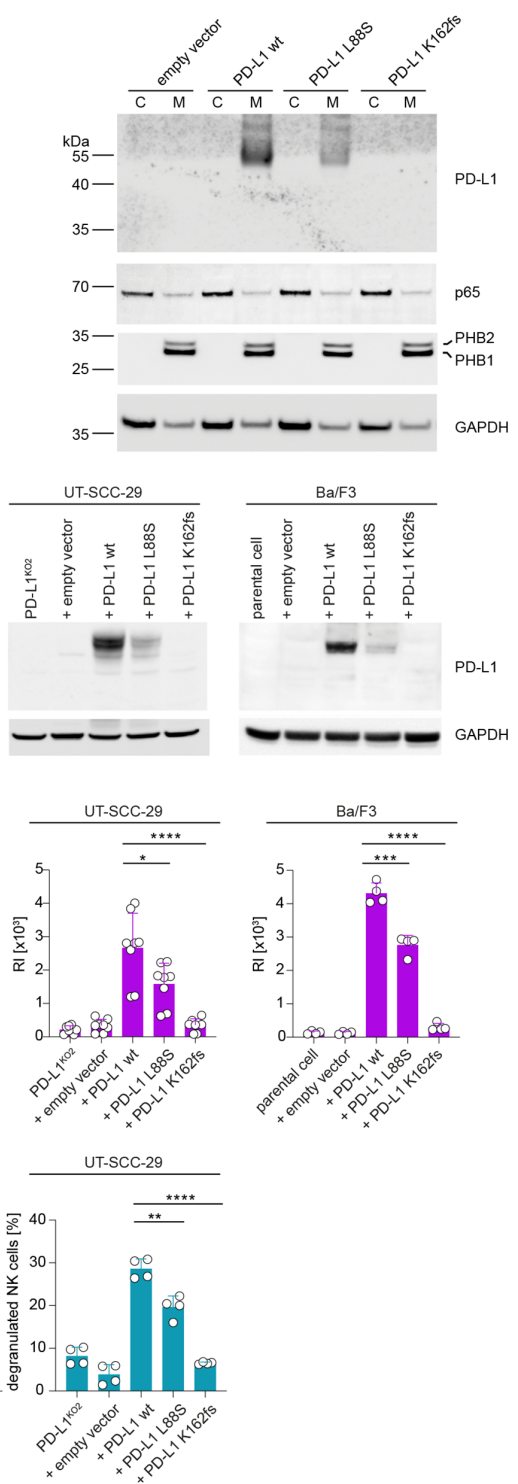

G
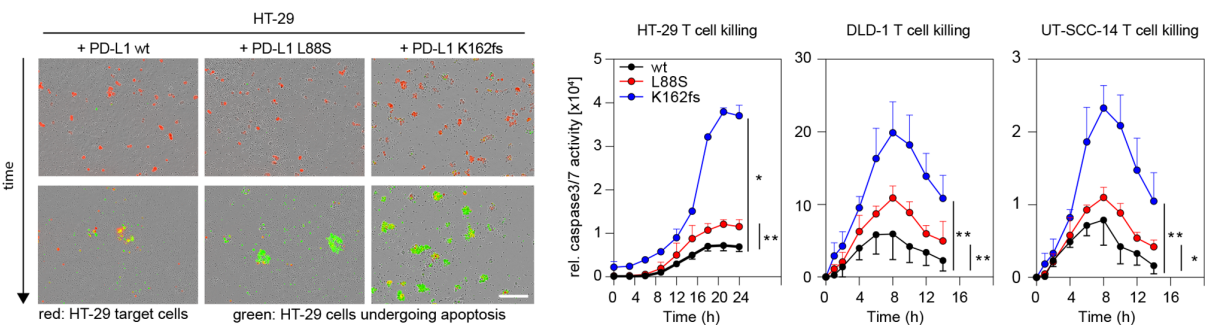

Figure 5 Selected PD-L1 mutations reduce protein abundancy, surface expression, ADCC and T cell suppression. (A) Schematic representation of gRNAs targeting the PD-L1 coding sequence (CDS). (B) CRISPR/Cas9-mediated depletion of PD$L 1$ as detected in crude cell extracts via immunoblotting. (C) PD-L1 protein levels after lentiviral redelivery of $P D-L 1$ variants into $P D-L 1^{K O 2}$ human cell lines and the murine $\mathrm{Ba} / \mathrm{F} 3$ cell line as detected by immunoblotting. (D) Enrichment of PD-L1 in the membranous fraction (M) of HT-29 cell variants from panel C. (C) Cytosolic, non-membranous fraction. (E) Flow cytometric detection of PD-L1 surface expression displayed as mean relative fluorescence intensity (RI) after staining with avelumab. $n(D L D-1)=7, n(H T-29)=8, n(U T-S C C-14)=9, n(U T-S C C-29)=8, n(B a / F 3)=4$. (F) NK cell degranulation induced by coculturing primary NK cells and cell lines expressing indicated $P D-L 1$ variants in the presence of avelumab ( $\mathrm{n}=4$ for DLD-1, HT-29, UTSCC-29; $n=5$ for UT-SCC-14). Percent degranulated NK cells normalized to spontaneous NK degranulation is shown for all cocultures. (G) Representative images and time course of T cell mediated tumor cell killing. CD8+ T cells were cocultured with HT-29, DLD-1 and UT-SCC-14 cells expressing WT PD-L1 or the L88S and K162fs variants (red fluorescence). Caspase 3/7 activity (relative intensity of green fluorescence) was monitored every $90 \mathrm{~min}$ for 24 hours with an Incuyte S3. Scale bar, $200 \mu \mathrm{M}$. Asterisks indicate $p$ value range $\left({ }^{*} p<0.05 ;{ }^{* *} p<0.01 ;{ }^{* *} p<0.001 ;{ }^{* * *} p<0.0001\right)$. Statistics for time course: two-tailed paired t test. All other statistics: two-tailed unpaired t test. NK, natural killer; PD-L1, programmed cell death protein ligand 1. 
A
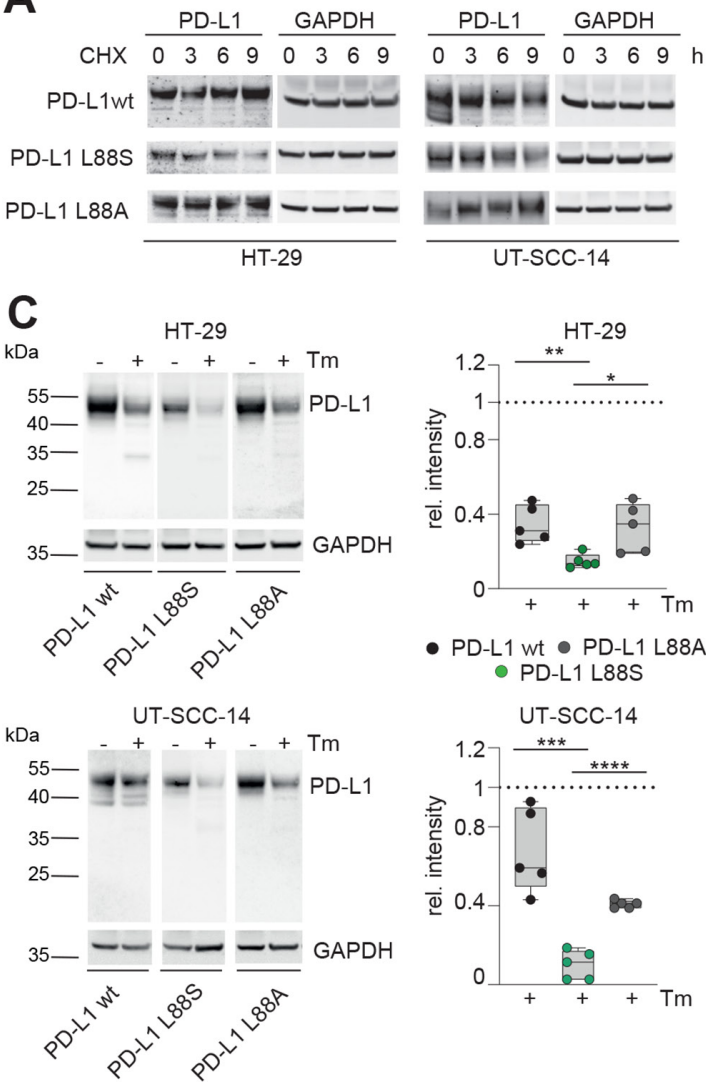

E glycosylated $(\bullet)$ non-glycosylated $(\bullet \bullet)$

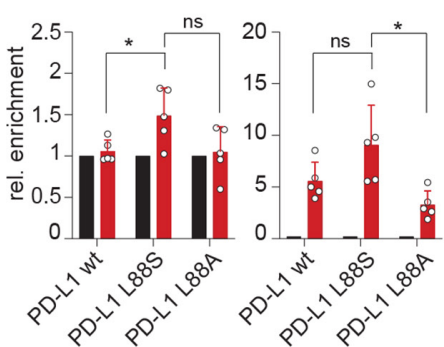

B

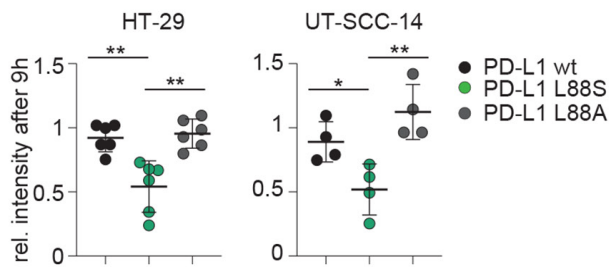

D

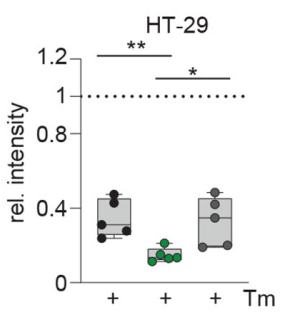

- PD-L1 wt • PD-L1 L88A - PD-L1 L88S

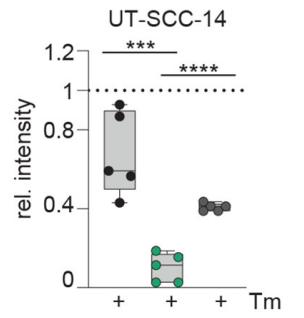

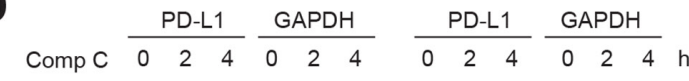
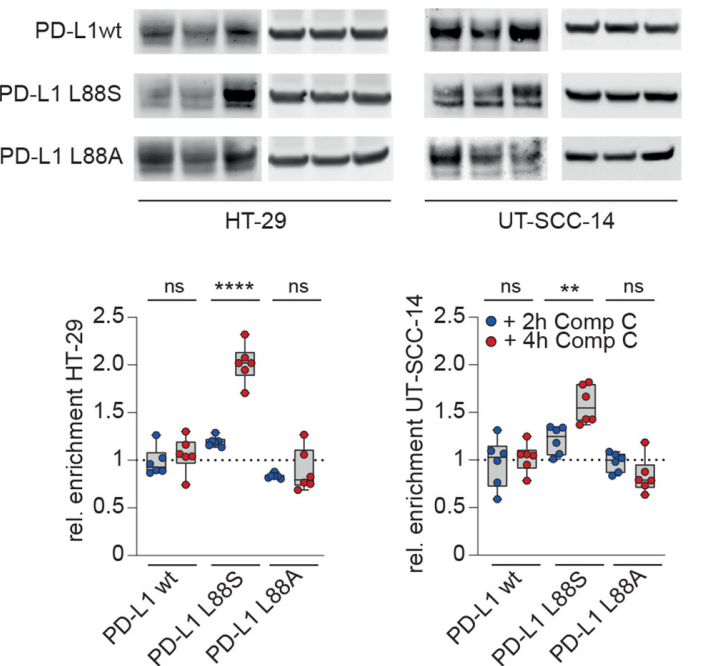

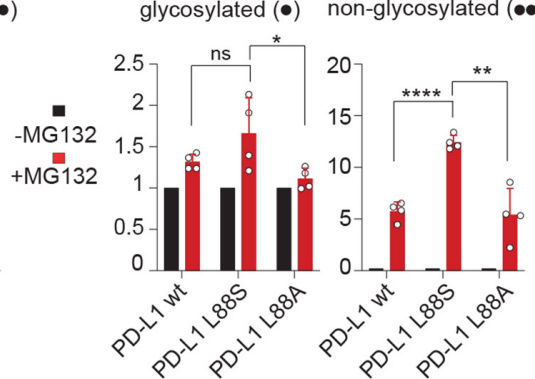

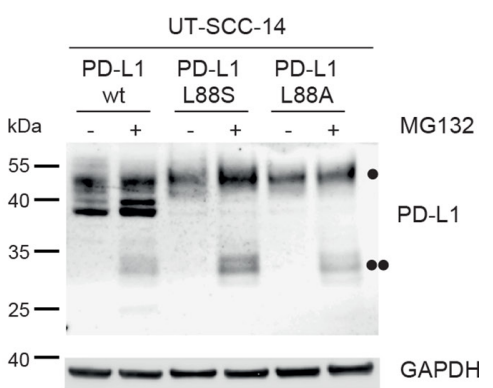

Figure 6 PD-L1 L88S exhibits enhanced phosphorylation-dependent proteasomal degradation. (A) immunoblot analysis of PD-L1 abundancy in HT-29 $(n=6)$ and UT-SCC-14 $(n=4)$ cells overexpressing PD-L1 variants after blocking protein synthesis with $20 \mu \mathrm{M} \mathrm{CHX}$ for 3, 6 and 9 hours (h). (B) Quantification of (A) and four replicates using ImageJ. (C) Relative stability of PD-L1 protein (=signal intensity relative to control as quantified using ImageJ) in PD-L1 overexpressing cells after abrogation of $\mathrm{N}$ glycosylation for 18 hours using tunicamycin (Tm) as determined by immunoblotting ( $n=5)$. (D) Quantification of PD-L1 protein abundancy in PD-L1 overexpressing cells after 2-hour and 4-hour blocking of AMPK with $10 \mu \mathrm{M}$ compound C (Comp C) as determined by immunoblotting $(n=6)$. (E) Enrichment of PD-L1 variants after inhibition of the proteasome using $20 \mu M$ MG132 for 4 hours. Quantification of glycosylated $(\bullet)$ and non-glycosylated $(\bullet \bullet)$ PD-L1 from four replicates of PD-L1 transduced HT-29 and UT-SCC-14 cells. Statistics: two-tailed unpaired t-test. Asterisks indicate $p$ value range $\left({ }^{*} p<0.05 ;{ }^{* *} p<0.01 ;{ }^{* *} p<0.001\right.$; $\left.{ }^{* * *} \mathrm{p}<0.0001 ; \mathrm{ns}>0.05\right)$. AMPK, AMP-activated protein kinase; PD-L1, programmed cell death protein ligand 1.

and consequently this mutation leads to full loss of PD-L1 on the cell surface. We experimentally showed that the loss of PD-L1 on the tumor cell surface induced by both type of mutations abrogated direct antitumor effects of avelumab (NK-mediated ADCC) while allowing enhanced T cell mediated tumor cell killing.

Together, the emergence of such mutations in patients expressing the $F c \gamma R 3 a$ V-allele demonstrates the selective pressure of avelumab in this subset. In addition to this general proof of concept, the nature of the detected mutations also provides insight into the mechanisms of action of this antibody. While loss of PD-L1 membrane expression represents a mechanism to evade direct tumor cell killing by avelumab, the loss of this antigen at the same time deprives the tumor of the chance to inactivate $T$ cells. This suggests that direct tumor cell killing by ADCC should be considered a clinically relevant mechanism of action of this antibody. Also, the association of the $F c \gamma R 3 a$ high-affinity SNP with PD-L1 mutations supported this interpretation. Very interestingly, recognized ICB 
resistance mutations co-occurred in one patient with a PD-L1 mutation (patient 22). This suggested that the reduction in membrane PD-L1 density allowing to evade avelumab tumor cell killing in this patient came at the price of higher selective pressure by T cells. Most likely, this $\mathrm{T}$ cell pressure ultimately led to selection of a tumor cell clone that also evaded $\mathrm{T}$ cell recognition by B2M loss and functional impairment of MHC class I antigen presentation. The fact that this patient had a MSI-H tumor suggests that 'uncompensated' loss of PD-L1 is likely less tolerated than in MSS tumors. Of note, the three patients with treatment-selected subclonal PD-L1 mutations showed above average PFS (18.1, 21.6 and 14.8 months), a finding that appeared somewhat counterintuitive at first glance since the mutations mediate immune escape to the direct antitumor effects of avelumab. Also, it appeared that in two cases, the mutations were present already many months before clinical resistance occurred: patient 22 showed the mutation in tumor tissue, which was Next-Generation Sequencing (NGS) detectable 10 months before clinical progression (by ddPCR even as a minimal tumor subclone before treatment initiation), and patient 21 showed a very low VAF of this mutation at week 9 on treatment (18 months before progression) before the clone finally achieved a mutant VAF of $3.1 \%$ at the time of progression. Together, the long PFS in patients that ultimately developed $P D-L 1$ mutations suggested that these were the patients in whom avelumab was active. The very slow selection dynamics of $P D-L 1$ mutations is also a strong indicator that other effects counteract this resistance mechanism (eg, more efficient $\mathrm{T}$ cell killing of PD-L1 loss tumor subclones) or that other components of the regimen may have efficiently suppressed these clones over a long time as observed for the RAS mutant clones in patients 5, 34, 12 and 42. Finally, the disappearance of these mutant subclones in two of two evaluable patients after avelumab withdrawal also points in the direction that these mutations reduce the overall biological fitness of the clone and therefore represent evolutionary dead-ends.

In summary, the addition of avelumab to cetuximab and chemotherapy was feasible and safe. The addition of the experimental ICB component did not result in clear signs of increased activity in this RAS/BRAF wildtype MSS mCRC cohort. Specific subpopulations-such as patients expressing the $F c \gamma R 3 a$ V-allele-showed a trend toward increased clinical benefit supported by the finding of subclonal PD-L1 escape mutations, which demonstrate the selective pressure exerted by this antibody. Future randomized trials should assess if addition of avelumab to standard of care (FOLFOX/FOLFIRI+EGFR/VEGF antibody) improves outcomes in patients using the $F c \gamma R 3 a$ V-allele. Given the selection dynamics of PD-L1 escape mutations and their disappearance after antibody withdrawal, such trials may incorporate a liquid biopsy-guided drug-holiday concept.

\section{Author affiliations}

${ }^{1}$ Hämatologisch-Onkologische Praxis Eppendorf, Hamburg, Germany ${ }^{2}$ Department of Oncology and Hematology, Bone Marrow Transplantation with Section Pneumology, University Medical Center Hamburg-Eppendorf, Hamburg, Germany

${ }^{3}$ Department of Internal Medicine IV - Oncology/Hematology, Martin-LutherUniversitat Halle-Wittenberg, Halle, Sachsen-Anhalt, Germany

${ }^{4}$ Department of Internal Medicine III (Haematology/Medical Oncology), Technical University of Munich Hospital Rechts der Isar, Munchen, Bayern, Germany

${ }^{5}$ University Hospital of Giessen and Marburg Campus Marburg, Marburg, Hessen, Germany

${ }^{6}$ Private Practice Onkodoc GmbH Gütersloh, Gütersloh, Nordrhein-Westfalen, Germany

${ }^{7}$ Department of Internal Medicine I, University Hospital UIm, Ulm, BadenWürttemberg, Germany

${ }^{8}$ Private Practice Onkozentrum Dresden, Dresden, Sachsen, Germany

${ }^{9}$ Institute of Clinical Cancer Research IKF at Northwest hospital, Frankfurt, Hessen, Germany

${ }^{10}$ Department of Hematology and Oncology, Munich Hospital Neuperlach, Munchen, Bayern, Germany

${ }^{11}$ Department of Hematology, Oncology and Tumorimmunology, Charite

Universitatsmedizin Berlin, Berlin, Germany

${ }^{12}$ IKF Klinische Krebsforschung GmbH at Krankenhaus Nordwest, Frankfurt, Hessen, Germany

${ }^{13}$ Clinical Cancer Research Consulting (CCRC), Düsseldorf, Germany

${ }^{14}$ Institute of Pathology, Martin Luther University Halle Wittenberg, Halle, SachsenAnhalt, Germany

${ }^{15}$ Institute of Medical Immunology, Martin-Luther-Universitat Halle-Wittenberg, Halle, Germany

${ }^{16}$ Institute of Pathology, University Hospital Halle, Halle, Germany

${ }^{17}$ Department of Oncology, Hematology and Bone Marrow Transplantation with Section Penumology, Hubertus Wald University Cancer Center, University Medical Center Hamburg-Eppendorf, Hamburg, Germany

\section{Twitter Donjete Simnica @DonjeteS and Mascha Binder @lab_binder}

Acknowledgements We would like to thank all patients and families as well as all participating study centers, the study team at the AIO Studien $\mathrm{gGmbH}$, namely Tobias Meyer and Wolfgang Hiegl at the Institute of Clinical Cancer Research, Krankenhaus Nordwest, Frankfurt, and the medical monitor at the University Cancer Center Hamburg Franziska Stein. Moreover, we would like to thank Malik Alawi and Michael Spohn from the UKE bioinformatics core facility for bioinformatical support, and Anja Haak for analysis of microsatellite status. We would also like to thank Alexander Navarrete-Santos and the cell sorting core facility at the University Hospital Halle (Saale) as well as Nadine Bley and the Core Facility Imaging at the Martin-Luther-University Halle (Saale).

Contributors Conceptualization: AS, EG and SHB, MBi; methodology: AS, DS, CS, LvW, LP, RS, MBa, CM, BS, CW and MBi; investigation: AS, DS, CS, LvW, LP, RS, $\mathrm{MBa}, \mathrm{CM}, \mathrm{BS}, \mathrm{CW}, \mathrm{MBi}, \mathrm{EG}$ and $\mathrm{SHB}$; formal analysis: $\mathrm{EW}$ and $\mathrm{AH}$; data curation: $\mathrm{LW}$; writing - original draft: AS, DS, CS, LvW, LP, RS, MBa, CW, MBi, EG, SHB and AH; writing - review and editing: AS, DS, CS and MBi; recources: JT, MS, SL, JKS, RD, TE, SD, SEA, MK, UP, CB, AS, EG and SHB; supervision: MBi, AS and SHB. All authors had access to the study data and reviewed and approved the final manuscript.

Funding The legal sponsor of the trial is the AlO Studien $\mathrm{gGmbH}$, Berlin Germany. Merck KGaA Darmstadt, Germany, as part of an alliance between Merck KGaA and Pfizer supported the trial with study medication and a research grant to the AIO.

Competing interests AS received institutional research grants from Merck, BMS, Roche, Sanofi, Servier and honoraria for lectures and advisory board meetings by Merck, Roche, Amgen, Lilly, Sanofi-Aventis, Servier, Bayer, BMS, MSD and Sirtex. S-E A-B has an advisory role with Merck, Roche, Celgene, Lilly, Nordic Pharma, Bristol-Myers Squibb, Astellas and MSD Sharp \& Dohme; is a speaker for Roche, Celgene, Lilly, Nordic Pharma, AlO gGmbH, MCl, promedicis, Forum für Medizinische Fortbildung and Taiho pharma; he is CEO/founder of IKF Klinische Krebsforschung $\mathrm{GmbH}$ at Northwest Hospital; and has received research grants from Sanofi, Merck, Roche, Celgene, Vifor, Medac, Hospira, Lilly, Eurozyto, German Cancer Aid (Krebshilfe), German Research Foundation and the Federal Ministry of Education and Research. UP received institutional research grants from Celgene, BMS, Amgen, Lilly, Roche, Sanofi and Servier and honoraria for lectures and advisory board meetings by Roche, Celgene, Amgen, Lilly, Sanofi-Aventis, Servier, Bayer and BMS. $\mathrm{AH}$ received honoraria for lectures from Roche. $\mathrm{CB}$ received institutional research 
grants and honoraria for lectures and advisory board meetings from Merck, BMS, Roche, Sanofi, Servier, Bayer, BMS, Astrazeneca, Lilly, Mundipharma, Hexal, MSD and GSO. MB received institutional research grants from Merck, BMS, Hexal, German Cancer Aid (Krebshilfe), German Research Foundation and the Federal Ministry of Education and Research as well as honoraria for lectures and advisory board meetings by Celgene, Janssen, Gilead, Merck, Roche, Amgen, Sanofi-Aventis and BMS.

\section{Patient consent for publication Not required}

Provenance and peer review Not commissioned; externally peer reviewed.

Data availability statement Data are available in a public, open access repository. All data relevant to the study are included in the article or uploaded as supplementary information. Data and materials availability: NGS fastq files are deposited at European Nucleotide Archive, ID: PRJEB35507.

Supplemental material This content has been supplied by the author(s). It has not been vetted by BMJ Publishing Group Limited (BMJ) and may not have been peer-reviewed. Any opinions or recommendations discussed are solely those of the author(s) and are not endorsed by BMJ. BMJ disclaims all liability and responsibility arising from any reliance placed on the content. Where the content includes any translated material, BMJ does not warrant the accuracy and reliability of the translations (including but not limited to local regulations, clinical guidelines, terminology, drug names and drug dosages), and is not responsible for any error and/or omissions arising from translation and adaptation or otherwise.

Open access This is an open access article distributed in accordance with the Creative Commons Attribution Non Commercial (CC BY-NC 4.0) license, which permits others to distribute, remix, adapt, build upon this work non-commercially, and license their derivative works on different terms, provided the original work is properly cited, appropriate credit is given, any changes made indicated, and the use is non-commercial. See http://creativecommons.org/licenses/by-nc/4.0/.

\section{ORCID iDs}

Donjete Simnica https://orcid.org/0000-0003-2286-1381

Christoph Schultheiß http://orcid.org/0000-0001-9789-5776

Rebekka Scholz http://orcid.org/0000-0003-2077-0718

Lisa Paschold https://orcid.org/0000-0003-0020-1315

Barbara Seliger http://orcid.org/0000-0002-5544-4958

Mascha Binder https://orcid.org/0000-0003-0663-3004

\section{REFERENCES}

1 Overman MJ, McDermott R, Leach JL, et al. Nivolumab in patients with metastatic DNA mismatch repair-deficient or microsatellite instability-high colorectal cancer (CheckMate 142): an open-label, multicentre, phase 2 study. Lancet Oncol 2017;18:1182-91.

2 Overman MJ, Lonardi S, Wong KYM, et al. Durable clinical benefit with nivolumab plus ipilimumab in DNA mismatch Repair-Deficient/ Microsatellite Instability-High metastatic colorectal cancer. J Clin Oncol 2018;36:773-9.

3 André T, Shiu K-K, Kim TW, et al. Pembrolizumab in MicrosatelliteInstability-High advanced colorectal cancer. $N$ Engl J Med 2020;383:2207-18.

4 Grothey A, Tabernero J, Arnold D. Fluoropyrimidine (FP) + bevacizumab (BEV) + atezolizumab vs FP/BEV in BRAFwt metastatic colorectal cancer (mCRC): findings from cohort 2 of MODUL. Ann Oncol supp/ 2018;29:714-5.

5 Le DT, Uram JN, Wang $\mathrm{H}$, et al. $\mathrm{Pd}-1$ blockade in tumors with mismatch-repair deficiency. N Engl J Med 2015;372:2509-20.

6 Eng C, Kim TW, Bendell J, et al. Atezolizumab with or without cobimetinib versus regorafenib in previously treated metastatic colorectal cancer (IMblaze370): a multicentre, open-label, phase 3, randomised, controlled trial. Lancet Oncol 2019;20:849-61.

7 Duffy AG, Greten TF. Immunological off-target effects of standard treatments in gastrointestinal cancers. Ann Oncol 2014;25:24-32.

8 Pozzi C, Cuomo A, Spadoni I, et al. The EGFR-specific antibody cetuximab combined with chemotherapy triggers immunogenic cell death. Nat Med 2016;22:624-31.

9 Tesniere A, Schlemmer F, Boige V, et al. Immunogenic death of colon cancer cells treated with oxaliplatin. Oncogene 2010;29:482-91.

10 Woolston A, Khan K, Spain G, et al. Genomic and transcriptomic determinants of therapy resistance and immune landscape evolution during anti-EGFR treatment in colorectal cancer. Cancer Cell 2019;36:35-50.

11 Boyerinas B, Jochems C, Fantini M, et al. Antibody-Dependent cellular cytotoxicity activity of a novel anti-PD-L1 antibody Avelumab
(MSB0010718C) on human tumor cells. Cancer Immunol Res 2015;3:1148-57.

12 Wickenhauser C, Bethmann D, Feng Z, et al. Multispectral fluorescence imaging allows for distinctive topographic assessment and subclassification of tumor-infiltrating and surrounding immune cells. Methods Mol Biol 2019;1913:13-31.

13 Akyüz N, Brandt A, Stein A, et al. T-Cell diversification reflects antigen selection in the blood of patients on immune checkpoint inhibition and may be exploited as liquid biopsy biomarker. Int $J$ Cancer 2017;140:2535-44.

14 Mährle T, Akyüz N, Fuchs $P$, et al. Deep sequencing of bone marrow microenvironments of patients with del $(5 q)$ myelodysplastic syndrome reveals imprints of antigenic selection as well as generation of novel T-cell clusters as a response pattern to lenalidomide. Haematologica 2019;104:2018.208223.

15 Mohme M, Schliffke S, Maire CL, et al. Immunophenotyping of newly diagnosed and recurrent glioblastoma defines distinct immune exhaustion profiles in peripheral and tumor-infiltrating lymphocytes. Clin Cancer Res 2018;24:4187-200.

16 Schliffke S, Akyüz N, Ford CT, et al. Clinical response to ibrutinib is accompanied by normalization of the T-cell environment in CLLrelated autoimmune cytopenia. Leukemia 2016;30:2232-4.

17 Schliffke S, Carambia A, Akyüz N, et al. T-Cell repertoire profiling by next-generation sequencing reveals tissue migration dynamics of TRBV13-family clonotypes in a common experimental autoimmune encephalomyelitis mouse model. J Neuroimmunol 2019;332:49-56.

18 Simnica D, Akyüz N, Schliffke S, et al. T cell receptor next-generation sequencing reveals cancer-associated repertoire metrics and reconstitution after chemotherapy in patients with hematological and solid tumors. Oncoimmunology 2019;8:e1644110.

19 Simnica D, Schliffke S, Schultheiß C, et al. High-Throughput immunogenetics reveals a lack of physiological T cell clusters in patients with autoimmune cytopenias. Front Immunol 2019;10:1897.

20 Bolotin DA, Poslavsky S, Mitrophanov I, et al. MiXCR: software for comprehensive adaptive immunity profiling. Nat Methods 2015;12:380-1.

21 R. C. Team. $R$ foundation for statistical computing. Vienna, Austria, 2018.

22 Nazarov VI, Pogorelyy MV, Komech EA, et al. tcR: an R package for T cell receptor repertoire advanced data analysis. BMC Bioinformatics 2015;16:175.

23 Cerami E, Gao J, Dogrusoz U, et al. The cBio cancer genomics portal: an open platform for exploring multidimensional cancer genomics data. Cancer Discov 2012;2:401-4.

24 Xu C, Gu X, Padmanabhan R, et al. smCounter2: an accurate lowfrequency variant caller for targeted sequencing data with unique molecular identifiers. Bioinformatics 2019;35:1299-309.

25 Tintelnot J, Baum N, Schultheiß C, et al. Nanobody targeting of epidermal growth factor receptor (EGFR) ectodomain variants overcomes resistance to therapeutic EGFR antibodies. Mol Cancer Ther 2019;18:823-33.

26 Alter G, Malenfant JM, Altfeld M. CD107a as a functional marker for the identification of natural killer cell activity. J Immunol Methods 2004;294:15-22.

27 Yamashita K, Iwatsuki M, Harada K, et al. Can PD-L1 expression evaluated by biopsy sample accurately reflect its expression in the whole tumour in gastric cancer? Br J Cancer 2019;121:278-80.

28 Li C, Huang C, Mok TS, et al. Comparison of 22C3 PD-L1 expression between surgically resected specimens and paired tissue microarrays in non-small cell lung cancer. $J$ Thorac Oncol 2017;12:1536-43.

29 Kvistborg P, Philips D, Kelderman S, et al. Anti-Ctla-4 therapy broadens the melanoma-reactive CD8+ T cell response. Sci Trans/ Med 2014;6:ra128.

30 Robert L, Tsoi J, Wang X, et al. Ctla4 blockade broadens the peripheral T-cell receptor repertoire. Clin Cancer Res 2014;20:2424-32.

31 Van Emburgh BO, Arena S, Siravegna G, et al. Acquired Ras or EGFR mutations and duration of response to EGFR blockade in colorectal cancer. Nat Commun 2016;7:13665.

32 Misale S, Yaeger R, Hobor S, et al. Emergence of KRAS mutations and acquired resistance to anti-EGFR therapy in colorectal cancer Nature 2012;486:532-6.

33 Diaz LA, Williams RT, Wu J, et al. The molecular evolution of acquired resistance to targeted EGFR blockade in colorectal cancers. Nature 2012;486:537-40.

34 Arena S, Bellosillo B, Siravegna G, et al. Emergence of multiple EGFR extracellular mutations during cetuximab treatment in colorectal cancer. Clin Cancer Res 2015;21:2157-66.

35 Montagut C, Dalmases A, Bellosillo B, et al. Identification of a mutation in the extracellular domain of the epidermal growth factor 
receptor conferring cetuximab resistance in colorectal cancer. Nat Med 2012;18:221-3.

36 Braig F, März M, Schieferdecker A, et al. Epidermal growth factor receptor mutation mediates cross-resistance to panitumumab and cetuximab in gastrointestinal cancer. Oncotarget 2015;6:12035-47.

37 Vidal J, Muinelo L, Dalmases A, et al. Plasma ctDNA Ras mutation analysis for the diagnosis and treatment monitoring of metastatic colorectal cancer patients. Ann Oncol 2017;28:1325-32.

38 Morelli MP, Overman MJ, Dasari A, et al. Characterizing the patterns of clonal selection in circulating tumor DNA from patients with colorectal cancer refractory to anti-EGFR treatment. Ann Oncol 2015;26:731-736.

39 Kim TW, Peeters M, Thomas A, et al. Impact of Emergent Circulating Tumor DNA RAS Mutation in Panitumumab-Treated Chemoresistant Metastatic Colorectal Cancer. Clin Cancer Res 2018;24:5602-5609.

40 Siravegna G, Mussolin B, Buscarino M, et al. Clonal evolution and resistance to EGFR blockade in the blood of colorectal cancer patients. Nat Med 2015;21:795-801.

41 Zaretsky JM, Garcia-Diaz A, Shin DS, et al. Mutations associated with acquired resistance to PD-1 blockade in melanoma. $N$ Engl J Med 2016;375:819-29.

42 Bibeau F, Lopez-Crapez E, Di Fiore F, et al. Impact of Fc\{gamma\} Rlla-Fc\{gamma\}RIlla polymorphisms and KRAS mutations on the clinical outcome of patients with metastatic colorectal cancer treated with cetuximab plus irinotecan. J Clin Oncol 2009;27:1122-9.

43 Trotta AM, Ottaiano A, Romano C, et al. Prospective evaluation of Cetuximab-Mediated antibody-dependent cell cytotoxicity in metastatic colorectal cancer patients predicts treatment efficacy. Cancer Immunol Res 2016;4:366-74.

44 European medicines Agency. (2017), vol. August 2019.

45 Li C-W, Lim S-O, Xia W, et al. Glycosylation and stabilization of programmed death ligand-1 suppresses T-cell activity. Nat Commun 2016;7:12632.

46 Cha J-H, Yang W-H, Xia W, et al. Metformin promotes antitumor immunity via endoplasmic-reticulum-associated degradation of PDL1. Mol Cell 2018;71:e607.

47 Bokemeyer C, Bondarenko I, Hartmann JT, et al. Efficacy according to biomarker status of cetuximab plus FOLFOX-4 as first-line treatment for metastatic colorectal cancer: the OPUS study. Ann Oncol 2011;22:1535-46.

48 Venook AP, Niedzwiecki D, Lenz H-J, et al. Effect of first-line chemotherapy combined with cetuximab or bevacizumab on overall survival in patients with KRAS wild-type advanced or metastatic colorectal cancer: a randomized clinical trial. JAMA 2017;317:2392-401.

49 Qin S, Li J, Wang L, et al. Efficacy and Tolerability of First-Line Cetuximab Plus Leucovorin, Fluorouracil, and Oxaliplatin (FOLFOX-4) Versus FOLFOX-4 in Patients With RAS Wild-Type Metastatic Colorectal Cancer: The Open-Label, Randomized, Phase III TAILOR Trial. J Clin Oncol 2018;36:JCO2018783183.

50 Innocenti F, Ou F-S, Qu X, et al. Mutational analysis of patients with colorectal cancer in CALGB/SWOG 80405 identifies new roles of microsatellite instability and tumor mutational burden for patient outcome. J Clin Oncol 2019;37:1217-27. 